\title{
Risk factors for relapse or persistence of bacteraemia caused by Enterobacter spp.: a case-control study
}

Patrick N. A. Harris ${ }^{1 *}$, Anna M. Peri ${ }^{2}$, Anita M. Pelecanos ${ }^{3}$, Carly M. Hughes ${ }^{4}$, David L. Paterson ${ }^{5}$ and John K. Ferguson $6,7,4$

\begin{abstract}
Background: Enterobacter spp. possess chromosomal AmpC beta-lactamases that may be expressed at high levels. Previous studies have demonstrated a risk of relapsed bacteraemia following therapy with third generation cephalosporins (3GCs). What additional factors predict microbiological failure in Enterobacter bacteraemia is unclear. We aimed to determine factors associated with microbiological failure in Enterobacter bacteraemia.

Methods: We retrospectively identified cases of bacteraemia caused by Enterobacter spp. occurring in four hospitals. Using a case-control design, we determined clinical risk factors for persistence or relapse defined as repeated positive blood cultures collected between 72 hours and up to 28 days post initial positive blood culture.

Results: During the study period a total of 922 bacteraemia events caused by Enterobacter spp. in adults were identified. The overall risk of relapsed or persisting bacteraemia at 28 days was low (31 of 922, 3.4\%), with only 2 patients experiencing emergent resistance to 3GCs. A total of 159 patients were included in the case-control study. Using multivariate logistic regression, independent predictors for relapse were a line-associated source of infection (OR 3.87; 95\% Cl 1.56-9.60, $p=0.004$ ) and the presence of immunosuppression (OR 2.70; 95\% Cl 1.14-6.44, $p=0.02$ ). On univariate analysis definitive therapy with a broad-spectrum beta-lactam-beta-lactamase inhibitor (BLBLI, e.g. piperacillin-tazobactam) was not associated with relapse (OR 1.83; 95\% Cl 0.64-5.21, $p=0.26$ ) although the proportion of patients receiving a BLBLI as definitive therapy was relatively small (21/159, 13.2\%).

Conclusions: The risk of relapsed or persistent Enterobacter bacteraemia appears to be low in Australia. A line-associated source of infection and immunocompromise were significant independent predictors for relapse. Larger, preferably randomized, studies are needed to address whether BLBLIs represent an effective carbapenem-sparing option for Enterobacter bacteraemia.
\end{abstract}

Keywords: Enterobacter cloacae, Enterobacter aerogenes, Beta-lactamase, AmpC, Bacteraemia, Outcomes, Relapse, Treatment

\footnotetext{
* Correspondence: p.harris@uq.edu.au

${ }^{1}$ University of Queensland, UQ Centre for Clinical Research, Royal Brisbane and Women's Hospital, Building 71/918 Royal Brisbane \& Women's Hospital Campus, 4029 Herston, QLD, Australia

Full list of author information is available at the end of the article
} 


\section{Background}

Bacteria belonging to the genus Enterobacter spp. are Gram-negative Proteobacteria of the family Enterobacteriaceae and currently comprise 22 species [1]. They represent a diverse group which are widely distributed in nature [2] and possess multiple mechanisms to allow survival in a variety of environmental niches [3]. In humans, they may cause a wide variety of clinical infections and are a common cause of bacteraemia [2], especially within adult and neonatal intensive care units (ICUs) $[4,5]$.

The annual incidence of bacteraemia caused by Enterobacter spp. has been reportedly increasing in some parts of the world [6]. This bacterial genus presents particular challenges for the selection of optimal therapy due to the presence of chromosomally encoded AmpC beta-lactamase enzymes [7]. These enzymes are able to hydrolyse many beta-lactams, including third generation cephalosporins and may be induced by beta-lactam exposure. Furthermore, AmpC gene expression can become constitutively de-repressed by mutational loss of regulatory genes, leading to high-levels of AmpC production and a phenotype that demonstrates in vitro resistance to most beta-lactams and beta-lactam/betalactamase inhibitor (BLBLI) combination agents, except cefepime and carbapenems. [2] Such variants are usually present at low levels (e.g. between $10^{-5}$ to $10^{-7}$ of the total bacterial population) but may be rapidly selected during antibiotic therapy [2]. This phenomenon has been best described with the use of third generation cephalosporins (3GCs). In a prospective study of Enterobacter bacteraemia, emergence of resistance developed during treatment in 6 of 31 (19\%) bacteraemia episodes treated with 3GCs [8]. Similar outcomes were replicated in a later study [9], although relapse rates have been reported as lower in other studies [10-12]. In a large cohort of patients from South Korea, emergent resistance during therapy with 3GCs was more likely to occur with a complex focus of infection (particularly malignant bile duct obstruction) but was never seen in urinary tract infections [10]. Development of resistance in Enterobacter infections has been associated with higher mortality and healthcare-related costs [13]. As a result, 3GCs are usually not recommended as first line therapy, even when susceptible in vitro.

Enterobacter may also acquire other major resistance determinants such as extended-spectrum beta-lactamase (ESBL) enzymes or carbapenemases [14, 15]. Carbapenem resistance may also develop from AmpChyperproduction in association with porin mutations [16] and multi-drug resistant (MDR) Enterobacter has been implicated in nosocomial outbreaks [17-21]. As such, Enterobacter spp. have become important 'problem' pathogens in the healthcare setting [22]. Most significant Enterobacter infections are treated with agents such as carbapenems, quinolones or aminoglycosides. Cefepime may be a useful option given its stability to AmpC enzymes [23]. Trimethoprim-sulphamethoxazole may also be effective [24], but is rarely used in contemporary practice for serious invasive Enterobacter infections. BLBLI agents, such as piperacillin-tazobactam, have an uncertain role in this context. [7] Although they are often avoided over concerns relating to the development of AmpC-mediated resistance, the risk of this occurring for this antibiotic class has rarely been examined, and may even be associated with improved outcome when used as empirical therapy in one study [25].

\section{Methods}

The aim of the study was to determine clinical factors associated with relapsed or persistent infection in patients with Enterobacter bacteraemia. A specific hypothesis to be tested was that the use of BLBLI agents as definitive therapy carried no additional risk of bacteraemia relapse when compared with alternative established therapies.

\section{Setting}

The study was undertaken across four hospitals served by two public microbiology laboratories in Queensland and New South Wales, Australia. These included the Royal Brisbane and Women's Hospital (in Queensland), John Hunter Hospital in Newcastle, The Mater Hospital in Newcastle and Belmont Hospital (all in New South Wales).

\section{Study design}

A case-control design was used to determine risk factors for bacteraemia relapse or persistence beyond 3 days following initial positive blood culture. Adult patients ( $>=18$ years of age) with laboratory confirmed Enterobacter spp. in at least one blood culture draw were identified from August 1998 to August 2012 from Pathology North, and between October 1999 and November 2015 from Pathology Queensland. 'Relapsed or persistent' cases were defined if there was any positive blood culture $\geq 72$ hours, and up to 28 days, after the initial positive blood culture with identification of an Enterobacter isolate of the same species. Control patients (at a ratio of approximately 1:4) were randomly selected from those patients who had no relapsed bacteraemia during the 28 days following initial positive blood culture. For the logistic regression, control patients who died within 28 days were excluded as it was unknown whether they would have relapsed had they survived.

\section{Inclusion / exclusion criteria}

Any patient with at least one positive monomicrobial blood culture with Enterobacter spp. was included. 
Patients aged $\leq 17$ years at the time of infection were excluded.

\section{Clinical data collection}

Cases were identified by data extraction from the Pathology North and Pathology Queensland laboratory information systems (AUSLAB; PJA, Melbourne). Clinical and demographic data on all significant bloodstream infections were prospectively collected as routine surveillance by the infection control and microbiology services. Relapsed cases were retrospectively identified from laboratory data. Outcomes were determined at 28 days post initial positive blood culture for all cases and controls.

In addition to demographic details, clinical variables recorded included source of infection, hospital location, co-morbid conditions, admitting clinical service, acquisition status of infection, initial antibiogram of the blood culture isolate (AmpC de-repressed phenotype), the presence of vascular access devices and the neutrophil count on the day of first positive blood culture. Data on antibiotic use and SAPS II physiology scores [26] (determined on the day of first positive blood culture) were recorded from clinical chart review. Only antimicrobial agents with Gram-negative activity were recorded, with those used within the first $48 \mathrm{~h}$ after initial blood culture defined as empirical use and those prescribed after blood culture results available defined as definitive use. Healthcare acquisition and source designation of the bacteraemia were categorised according to standard definitions [27-29]. Empirical antibiotic therapy was described as appropriate if the isolate was susceptible to at least one agent used. If an agent was used for $\geq 50 \%$ of the definitive treatment duration, this was listed as the primary agent. If a second agent was used either concurrently or sequentially, the patient was described as receiving combination therapy; if combination therapy was used for the majority of the definitive treatment duration the antibiotic choice was determined as 'other'. For statistical analysis, if the definitive regimen included a carbapenem, quinolone, co-trimoxazole, cefepime or an aminoglycoside to which the isolate was susceptible, the treatment was classified as 'standard therapy', if piperacillin-tazobactam or ticarcillin-clavulanate was used and the isolate was susceptible, the treatment was classified as 'BLBLI', otherwise the treatment was defined as 'inappropriate' (e.g. cephalexin, cephazolin, ceftriaxone, ampicillin/amoxicillin, amoxicillin-clavulanate or no therapy). Standard dosing regimens at participating hospitals for piperacillin-tazobactam are $4.5 \mathrm{~g}$ 8-hourly and ticarcillin-clavulanate $3.1 \mathrm{~g}$ 6-hourly, with dose adjustment for renal dysfunction according to the Australian Therapeutic Guidelines [30]. Patients were classified as being immunosuppressed if they had the following conditions: neutropenia (neutrophil count $<0.5 \times 10^{9} / \mathrm{L}$ ), haematological / solid organ malignancy or myelodysplastic syndrome, solid organ transplant or if they received any other immunosuppressive drug therapy including prolonged high dose corticosteroids $(\geq 30 \mathrm{mg}$ prednisolone or equivalent daily).

\section{Microbiological methods}

Bacterial isolates were identified by routine diagnostic methods employed in the laboratory over the study period. Bacteraemia was diagnosed using the BD BACTEC automated system (Becton Dickinson, Sydney, Australia). Species identification methods used included the Vitek 2 or API 20E systems (Biomerieux, MarcyL'Etoile, France), BBL Crystal (Becton Dickinson), as well as MALDI-TOF (Vitek MS, Biomerieux) and routine bench testing. Susceptibility testing was performed using Kirby-Bauer disk diffusion methods or by Vitek2 automated microbroth dilution (Biomerieux), with interpretative standards as defined by the Clinical and Laboratory Standards Institute (CLSI) at the time of testing [31], although from 2012 onwards Pathology Queensland switched to EUCAST methodology [32].

\section{Statistical methods}

Data describing patient demographics, microbiology results, clinical features, bacteraemia source, co-morbidity, acquisition status and clinical service for all patients were tabulated for relapse cases and non-relapse cases, with categorical variables expressed as percentages and median (interquartile range [IQR]), mean (standard deviation $[\mathrm{SD}]$ ) and/or ranges calculated as appropriate for scale variables. Wilcoxon rank-sum test was used to explore differences between non-parametric continuous variables. Potential risk factors for relapse as the dependent variable were included in a univariate logistic regression model. Variables with a $p$-value of $<0.2$ and/or with large effect estimates (odds ratios $[\mathrm{OR}]>2$ or $<0.5$ ) in the univariate analysis were included in the multivariate model. Odds ratios with 95\% confidence intervals (CI) were calculated for predictors of relapse. The multivariate model was optimized using a stepwise approach, beginning with the univariate model demonstrating the strongest association with relapse. The goodness-of-fit of the model before and after each step was compared by the likelihood-ratio test and the optimal model fit determined using Akaike's and Bayesian information criteria. Variables that did not significantly improve the model fit were discarded. Statistical analysis was performed using Stata 13.1 (StataCorp; TX, USA). A $p$-value $<0.05$ was considered significant. Using a ratio of 1:4 cases to controls, and assuming a $40 \%$ exposure to variables in the control group that may influence outcome, we calculated that we would need 35 cases and 137 controls, 
assuming a two-sided confidence level of 95\% to achieve $80 \%$ power to detect an odds ratio of at least 3.0.

\section{Results}

During the study periods for each location, a total of 922 positive blood cultures growing Enterobacter spp. were identified, after excluding polymicrobial infections, individuals aged $<18$ years and repeatedly positive cultures within the first $72 \mathrm{~h}$ after initial bacteraemia. A total of $31(3.4 \%)$ patients were classified as having persistent or relapsed Enterobacter bacteraemia, occurring between 3 and 28 days post initial positive blood culture. A total of 159 patients (all 31 cases and 128 randomly-selected controls) were included in the case-control study to identify risk factors for bacteraemia relapse or persistence (see Fig. 1). The proportions of different species identified and their antimicrobial susceptibilities are shown in Table 1. The most common species identified was Enterobacter cloacae (76.7\%). A proportion of isolates had an initial antibiogram suggesting AmpC derepression $(61 / 159,38.4 \%)$, but of these only 9 relapsed. Furthermore, in only 3 of the relapsed cases did 3GC resistance develop during treatment $(1.9 \%$ of the total cohort) (see Additional file 1: Table S1 for detail).

Empirical combination therapy was common (73/159, $45.9 \%)$, most frequently employing ampicillin plus gentamicin (10/73, 13.7\%). Excluding patients treated with combination therapy, the most commonly prescribed agents for empirical treatment, with in vitro activity against Enterobacter spp., were aminoglycosides (31/86, 36\%) or a broad-spectrum BLBLI (ticarcillin-clavulanate or piperacillin-tazobactam) (12/86, 13.9\%) followed by carbapenems (11/86, 12.8\%). For definitive treatment, a carbapenem was most commonly used (45/ $159,28.3 \%)$, followed by a fluoroquinolone (39/159, 24.5\%), BLBLIs $(21 / 159,13.2 \%)$ and aminoglycosides $(11 / 159,6.9 \%)$ (Table 2). The median duration of treatment was 10 days (range 0 to 328 days; IQR 2-40 days) and did not differ significantly between cases and controls ( $p=0.13$, Wilcoxon rank-sum test). The mean SAPSII score was 34.7 (range 6-89; SD 13.6), which corresponds to a risk of in-hospital mortality of approximately $16.2 \%$.

Results for the univariate and multivariate logistic regression are summarised in Table 3. In the final model, the strongest predictors of relapsed or persistent bacteraemia were the presence of immunosuppression (OR 2.70; 95\% CI 1.14-6.44 $p=0.02$ ) or a lineassociated source of bacteraemia (OR 3.87; 95\% CI 1.56-9.60, $p=0.004$ ), whereas choice of definitive antibiotic therapy did not show a significant effect on the univariate analysis.

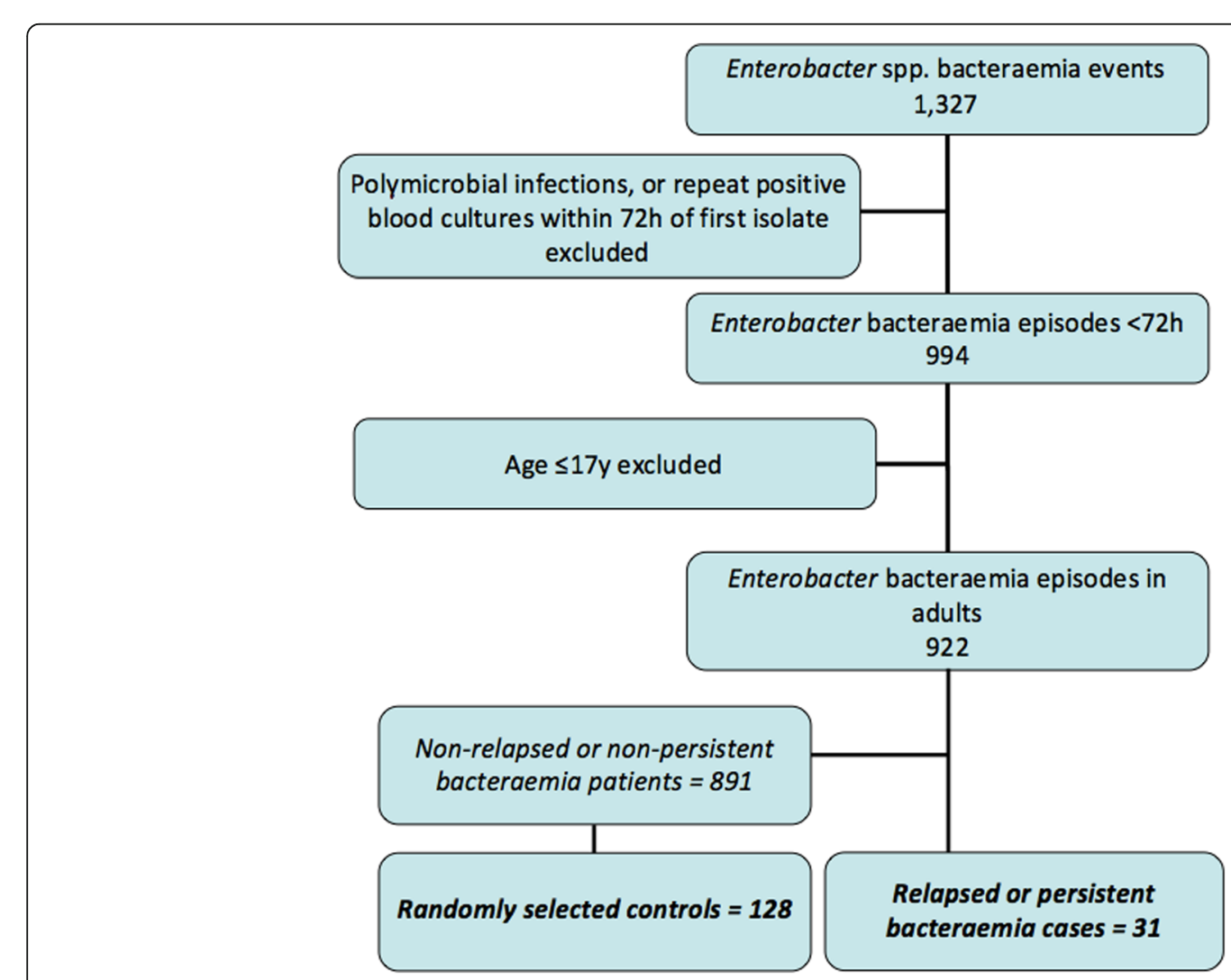

Fig. 1 Inclusion flowchart for case-control study 
Table 1 Antibiotic susceptibility of Enterobacter species causing bacteraemia

\begin{tabular}{|c|c|c|c|c|c|c|c|c|c|c|c|}
\hline \multirow[t]{2}{*}{ Species } & \multirow{2}{*}{$\begin{array}{l}\% \\
\text { Isolates } \\
\text { (Number) }\end{array}$} & \multicolumn{10}{|c|}{$\%$ Susceptibility (number tested) } \\
\hline & & $\mathrm{CRO}$ & GENT & TZP & TIM & CAZ & FEP & SXT & CIP & IMl & MER \\
\hline \multirow[t]{2}{*}{ Enterobacter cloacae } & 76.7 & 65.6 & 81.1 & 77.8 & 60.7 & 57.6 & 94.8 & 74.3 & 94.3 & 100 & 98.1 \\
\hline & (122) & $(122)$ & $(122)$ & (63) & $(107)$ & (85) & (96) & $(113)$ & $(122)$ & (41) & (105) \\
\hline \multirow[t]{2}{*}{ Enterobacter aerogenes } & 19.5 & 74.2 & 100 & 76.9 & 78.6 & 88.2 & 100 & 100 & 96.8 & 100 & 100 \\
\hline & $(31)$ & (31) & (31) & (13) & (28) & (17) & (27) & $(29)$ & (31) & (8) & (29) \\
\hline \multirow[t]{2}{*}{ Other Enterobacter spp. } & 3.8 & 66.7 & 83.3 & 100 & 75 & 50 & 100 & 83.3 & 100 & 100 & 80 \\
\hline & (6) & (6) & (6) & (3) & (4) & (4) & (4) & (6) & (6) & (2) & (5) \\
\hline \multirow[t]{2}{*}{ All species } & 100 & 67.3 & 84.9 & 78.5 & 64.7 & 62.3 & 96.1 & 79.7 & 95 & 100 & 97.8 \\
\hline & (159) & (159) & (159) & $\left(79^{a}\right)$ & (139) & (106) & (127) & (148) & (159) & (51) & (139) \\
\hline
\end{tabular}

CRO ceftriaxone, GENT gentamicin, TZP piperacillin-tazobactam, TIM ticarcillin-clavulanate, CAZ ceftazidime, FEP cefepime, CIP ciprofloxacin, MER meropenem, IMI imipenem, SXT trimethoprim-sulphamethoxazole

a Limited number of isolates as not routinely tested in all labs until 2010

\section{Discussion}

In this case-control study, 3.4\% of patients with Enterobacter bacteraemia were found to have relapsed by 28 days. The most striking finding is the effect of bacteraemia source on the risk of this outcome; $74 \%$ of all relapsed cases had a line-associated source for the bacteraemia, and this variable had the greatest effect size in the multivariate logistic regression model (OR 3.87). Although data were not available to reliably determine the precise date of line-removal in the majority of cases, we would hypothesise delayed line removal as a likely reason for this association.

Given the well-described association with $3 \mathrm{GC}$ treatment and AmpC-mediated relapse when used for Enterobacter infections, this study also aimed to assess the effect of antibiotic choice on the risk of relapse. When compared with alternative effective therapies (such as carbapenems, cefepime, quinolones or aminoglycosides) BLBLIs were not significantly associated with bacteraemia relapse when used as definitive therapy. However, BLBLIs were used relatively infrequently for definitive therapy. Overall antibiotic use was heterogeneous and often included combination therapy, meaning that effects for individual agents were diluted. We defined BLBLI therapy as including ticarcillin-clavulanate and piperacillin-tazobactam. However, these agents have some important differences with respect to AmpC-enzymes. Clavulanate is a potent inducer of $\mathrm{AmpC}$ and a poor inhibitor, whereas piperacillin and tazobactam are only weak inducers of AmpC [33].

Many laboratories suppress susceptibility results for AmpC-producers (such as Enterobacter spp.) in favour of carbapenems or quinolones due to concerns of selecting for AmpC de-repressed variants. According to the findings of this study, this may not be justified as a routine policy, especially in cases where source control has been addressed or in the absence of significant immunosuppression. If BLBLIs are used against susceptible

Table 2 Frequency of bacteraemia relapse by definitive treatment category

\begin{tabular}{llll}
\hline Definitive treatment category & Relapse (cases) & Non-relapse (controls) & Total \\
\hline Carbapenem & $8(25.8 \%)$ & $37(28.9 \%)$ & $45(28.3 \%)$ \\
Quinolones & $4(12.9 \%)$ & $35(27.3 \%)$ & $39(24.5 \%)$ \\
Other $^{a}$ & $6(19.4 \%)$ & $21(16.4 \%)$ & $27(17.0 \%)$ \\
Piperacillin-tazobactam/ticarcillin-clavulanate $_{\text {Aminoglycosides }}$ & $6(19.4 \%)$ & $15(11.7 \%)$ & $21(13.2 \%)$ \\
No antibiotic treatment & $3(9.7 \%)$ & $8(6.2 \%)$ & $11(6.9 \%)$ \\
Trimethoprim/sulphamethoxazole & $2(6.5 \%)$ & $2(1.6 \%)$ & $4(2.5 \%)$ \\
Cefepime & $1(3.2 \%)$ & $3(2.3 \%)$ & $4(2.5 \%)$ \\
Ampicillin/amoxicillin & $1(3.2 \%)$ & $2(1.6 \%)$ & $3(1.9 \%)$ \\
Cephazolin & $0(0.0 \%)$ & $2(1.6 \%)$ & $2(1.3 \%)$ \\
Ceftriaxone/ceftazidime & $0(0.0 \%)$ & $1(0.8 \%)$ & $1(0.6 \%)$ \\
Trimethoprim & $0(0.0 \%)$ & $1(0.8 \%)$ & $1(0.6 \%)$ \\
Total & $0(0.0 \%)$ & $1(0.8 \%)$ & $1(0.6 \%)$ \\
\hline
\end{tabular}

Includes combination therapy 
Table 3 Univariate and multivariate logistic regression of the effects of clinical variables on relapsed Enterobacter bacteraemia

\begin{tabular}{|c|c|c|c|c|c|c|c|c|c|}
\hline \multirow[t]{2}{*}{ Variable } & & \multirow{2}{*}{$\begin{array}{l}\text { Cases } \\
\text { (relapse) } \\
\mathrm{N}=31\end{array}$} & \multirow{2}{*}{$\begin{array}{l}\text { Controls } \\
\text { (non- } \\
\text { relapse) } \\
\mathrm{N}=128\end{array}$} & \multicolumn{3}{|c|}{ Univariate model } & \multicolumn{3}{|c|}{ Multivariate model } \\
\hline & & & & OR & $95 \% \mathrm{Cl}$ & $P$ & OR & $95 \% \mathrm{Cl}$ & $P$ \\
\hline \multicolumn{2}{|l|}{ Age, mean (SD) } & $52.0(17.0)$ & $60.5(18.6)$ & 0.98 & $0.95-1.00$ & \multicolumn{4}{|l|}{$0.02^{*}$} \\
\hline \multirow[t]{2}{*}{ Sex } & Female & $16(51.6 \%)$ & 49 (38.3\%) & \multicolumn{6}{|c|}{ female as reference } \\
\hline & Male & 15 (48.4\%) & $79(61.7 \%)$ & 0.58 & $0.26-1.28$ & \multicolumn{4}{|l|}{0.18} \\
\hline \multirow[t]{3}{*}{ Medical Service } & Med/surg & $10(32.3 \%)$ & $82(64.1 \%)$ & \multicolumn{6}{|c|}{ general medical / surgical as reference } \\
\hline & Haem/onc & 15 (48.4\%) & $30(23.4 \%)$ & 4.1 & $1.66-10.11$ & \multicolumn{4}{|l|}{$0.002^{*}$} \\
\hline & Renal & $6(19.4 \%)$ & $16(12.5 \%)$ & 3.08 & $0.98-9.66$ & \multicolumn{4}{|l|}{0.06} \\
\hline \multirow[t]{3}{*}{ Definitive therapy } & Standard & $23(74.2 \%)$ & $105(82.0 \%)$ & \multicolumn{6}{|c|}{$\begin{array}{l}\text { carbapenem, quinolone, cefepime, aminoglycoside or co-trimoxazole } \\
\text { as reference }\end{array}$} \\
\hline & BLBLI & $6(19.4 \%)$ & $15(11.7 \%)$ & 1.83 & $0.64-5.21$ & 0.26 & & & \\
\hline & Inappropriate & $2(6.5 \%)$ & $8(6.2 \%)$ & 1.14 & $0.23-5.73$ & 0.87 & & & \\
\hline \multirow[t]{2}{*}{ Source } & Non-line source & $8(25.8 \%)$ & $80(62.5 \%)$ & \multicolumn{6}{|c|}{ non-line source as reference } \\
\hline & Line-associated & $23(74.2 \%)$ & $48(37.5 \%)$ & 4.79 & $1.99-11.56$ & $<0.001^{*}$ & 3.87 & $1.56-9.60$ & $0.004^{*}$ \\
\hline \multirow{2}{*}{$\begin{array}{l}\text { Immune } \\
\text { suppression }\end{array}$} & Absent & $12(38.7 \%)$ & $83(64.8 \%)$ & \multicolumn{6}{|c|}{ no immunosuppression as reference } \\
\hline & Present & $19(61.3 \%)$ & $45(35.2 \%)$ & 2.92 & $1.30-6.56$ & $0.009^{*}$ & 2.70 & $1.14-6.44$ & $0.02^{*}$ \\
\hline \multirow[t]{2}{*}{ ICU admission } & Non-ICU & $25(80.6 \%)$ & $99(78.6 \%)$ & \multicolumn{6}{|c|}{ non-ICU admitted as reference } \\
\hline & ICU & $6(19.4 \%)$ & $27(21.4 \%)$ & 0.88 & $0.33-2.36$ & 0.80 & & & \\
\hline \multirow[t]{2}{*}{ Acquisition status } & Community & $2(6.5 \%)$ & $29(22.7 \%)$ & \multicolumn{6}{|c|}{ community-associated infection as reference } \\
\hline & Healthcare & $29(93.5 \%)$ & 99 (77.3\%) & 4.25 & 0.96-18.87 & 0.06 & & & \\
\hline \multirow[t]{2}{*}{ Region } & NSW & $15(48.4 \%)$ & $73(57.0 \%)$ & \multicolumn{6}{|c|}{ New South Wales region as reference } \\
\hline & Queensland & $16(51.6 \%)$ & $55(43.0 \%)$ & 1.42 & $0.64-3.11$ & 0.39 & & & \\
\hline \multirow[t]{2}{*}{ De-repressed AmpC phenotype } & Absent & $22(71.0 \%)$ & $76(59.4 \%)$ & \multicolumn{6}{|c|}{ no de-repressed AmpC as reference } \\
\hline & Present & $9(29.0 \%)$ & $52(40.6 \%)$ & 0.60 & $0.26-1.40$ & 0.24 & & & \\
\hline SAPS II score, mean (SD) & & $37.9(15.9)$ & $33.9(12.9)$ & 1.02 & $0.99-1.05$ & 0.14 & & & \\
\hline \multirow[t]{2}{*}{ Enterobacter species } & Other Enterobacter spp. & $6(19.4 \%)$ & $31(24.2 \%)$ & \multicolumn{6}{|c|}{ other Enterobacter spp. as reference } \\
\hline & E. cloacae & $25(80.6 \%)$ & $97(75.8 \%)$ & 1.33 & $0.50-3.54$ & 0.57 & & & \\
\hline
\end{tabular}

*significant $p<0.05$

Enterobacter isolates there does not appear to be any clear association with microbiological failure; and failure with the emergence of AmpC-mediated resistance appears to be rare. However, given the small number of patients treated with BLBLIs in this study, the possibility of a type II error exists, and this needs further investigation in larger cohorts. The proportion of patients treated with BLBLIs was lower than expected, which compromised the power of the study to address the effect of antibiotic choice on outcome.

In this current era of emerging carbapenem-resistance, it is critical that alternatives to carbapenems are sought for common infections. Infectious disease practitioners in our region most frequently recommend carbapenems for AmpC beta-lactamase producing Enterobacteriaceae [34]. As such, it may be a significant driver of carbapenem use. Our findings would suggest that using a BLBLI when susceptibility is demonstrated, especially in immunocompetent patients in whom adequate source control has been achieved, is not clearly associated with microbiological failure. Further work would help to delineate patients for whom carbapenems may still be a superior choice.

Not only was the risk of relapsed Enterobacter bacteraemia low (3.4\%) in this study, but in only 3 cases did resistance to 3GCs (a marker for AmpC de-repression) emerge during treatment. This figure is lower than described in some previous studies $[8,9]$. There may be several reasons for this observed difference. Firstly, clinicians are more aware of this clinical phenomenon and $3 \mathrm{GCs}$ are currently rarely used as definitive treatment for Enterobacter bacteraemia. In this study only 3 of 159 (1.9\%) bacteraemia events received either ceftriaxone or ceftazidime as definitive therapy, and in 2 of these 3GCs were given in combination with another agent. This may also reflect local laboratory practice for withholding susceptibility reporting for 3GCs against Enterobacter. Secondly, low rates of relapse may also result from 
better recognition of source control and the availability of other effective antibiotics, especially carbapenems, cefepime and fluoroquinolones. Other variables that may have influenced the low rate of relapse in this study might include the clinical characteristics of our patient population as well as long-standing infection control and antimicrobial stewardship programs.

The overall risk of Enterobacter bacteraemia with a de-repressed AmpC phenotype in our region was also relatively low over the study period, with only $38.4 \%$ of isolates being resistant to 3GCs (either ceftriaxone or ceftazidime). Very few isolates showed resistance to carbapenems, although MIC breakpoints were lowered during the study period. Both current and older clinical breakpoints may fail to detect the presence of carbapenemase in Enterobacteriaceae [35, 36]. Our findings may not be generalizable to populations encountering higher rates of baseline 3GC resistance, or frequent ESBL / carbapenemase acquisition in Enterobacter species.

Limitations of the study are acknowledged. Although the bacteraemia database was collected prospectively as part of routine surveillance, clinical and microbiological data extraction for Enterobacter cases was retrospective. Although a laboratory servicing several hospitals was used to identify cases, these occurred within a limited area in Australia and may not represent the diversity of infections and patient populations found in other states and countries. Whether all repeat bacteraemia episodes were true relapsed events or new infections is also not known, as isolates were not available for molecular typing. Equally, the presence of co-existing ESBLs was not sought systematically for isolates demonstrating resistance to 3GCs; inferring Amp-C de-repression based on the antibiogram alone may be unreliable. This study only examined relapsed bacteraemia, since such an endpoint is easily defined. However, the risk of relapsed infection and emergent resistance from other clinical sites infected with Enterobacter may be different and care should be taken when extrapolating from bacteraemia data alone.

Given the inherent limitations of retrospective studies, it is hoped that the efficacy of BLBLIs or other carbapenemsparing options for Enterobacter infections could be addressed in large prospective observational studies or even randomised controlled trials (RCTs). A pilot RCT comparing piperacillin-tazobactam with meropenem for bloodstream infections caused AmpC-producers is currently recruiting (MERINO-2 Trial; ClinicalTrials.gov identifier: NCT02437045).

\section{Conclusions}

In a cohort of patients with bacteraemia caused by Enterobacter species, the overall risk of relapsed bacteraemia was low. Patient factors, such as a line-source for the bacteraemia or the presence of immunosuppression, were the strongest predictors of relapse. Use of a BLBLI agent as definitive therapy was not associated with treatment failure, but the total number of patients treated with a BLBLI was relatively low. Although this would suggest that susceptibility in vitro should translate into in vivo efficacy for BLBLI agents, especially if the patient has adequate source control, further studies are warranted to determine if BLBLIs are a safe and effective carbapenem-sparing option, especially for patients with severe disease, a complex focus of infection or immune compromise. Our data also reinforce the importance of line removal when suspected as the source for bacteraemia, especially for isolates with the genetic capacity to respond rapidly to antimicrobial exposure.

\section{Additional file}

Additional file 1: Table S1. Clinical details of relapsed Enterobacter bacteraemia cases with emergence of antibiotic resistance. (DOCX 17 kb)

\section{Abbreviations}

3GC: Third generation cephalosporin; BLBLI: Beta-lactam/beta-lactamase inhibitor; CLSI: Clinical and laboratory standards institute; ESBL: Extendedspectrum beta-lactamase; EUCAST: The European committee on antimicrobial susceptibility testing; ICU: Intensive care unit; MALDI-TOF: Matrix assisted laser desorption/ionization time of flight; MDR: Multi-drug resistant; MIC: Mimimum inhibitory concentration; OR: Odds ratio; RCT: Randomised controlled trial

\section{Acknowledgements}

We would also like to acknowledge the contribution of Dr Stuart Paynter.

\section{Funding}

No external funding was required for this study. $\mathrm{PH}$ is supported by an Australian Postgraduate Award from the University of Queensland.

\section{Availability of data and materials}

The dataset generated during the current study are available at Harvard Dataverse: http://dx.doi.org/10.7910/DVN/56NCVU.

\section{Authors' contributions}

$\mathrm{PH}, \mathrm{DP}$ and JF conceived and designed the study. PH, JF, and $\mathrm{CH}$ were responsible for data collection, $\mathrm{PH}$ and $\mathrm{AP}$ performed the statistical analysis. All authors contributed to the writing of the manuscript and approved the final version

\section{Competing interests}

DP has received research grants from AstraZeneca and has attended Advisory Boards, acted as a consultant to, or given lectures with honoraria from Cubist Pharmaceuticals, Merck, AstraZeneca, SanofiAventis, Pfizer, Johnson \& Johnson and Leo Pharmaceuticals. All other authors declare no conflicts of interest.

Consent for publication

Not applicable.

Ethics approval and consent to participate

Ethical approval for the study, with a waiver for consent, was provided by the Royal Brisbane \& Women's Hospital Human Research Ethics Committee. (HREC/15/QRBW/599).

\section{Author details}

'University of Queensland, UQ Centre for Clinical Research, Royal Brisbane and Women's Hospital, Building 71/918 Royal Brisbane \& Women's Hospital Campus, 4029 Herston, QLD, Australia. ${ }^{2}$ Department of Biomedical and Clinical Sciences Luigi Sacco, University of Milan, Milan, Italy. ${ }^{3} \mathrm{QIMR}$ Berghofer Medical Research Institute, Herston, QLD, Australia. ${ }^{4}$ Pathology North - 
Hunter, John Hunter Hospital, Newcastle, NSW, Australia. ${ }^{5}$ UQ Centre for Clinical Research, Royal Brisbane and Women's Hospital, QLD, Australia \& Wesley Medical Research, University of Queensland, Toowong, QLD, Australia. ${ }^{6}$ School of Biomedical Sciences and Pharmacy, University of Newcastle, Newcastle, NSW, Australia. ${ }^{7}$ School of Rural Medicine, University of New England, Armidale, NSW, Australia.

Received: 26 November 2016 Accepted: 18 January 2017

Published online: 21 January 2017

\section{References}

1. Mezzatesta ML, Gona F, Stefani S. Enterobacter cloacae complex: clinical impact and emerging antibiotic resistance. Future Microbiol. 2012;7:887-902.

2. Sanders Jr WE, Sanders CC. Enterobacter spp.: pathogens poised to flourish at the turn of the century. Clin Microbiol Rev. 1997;10:220-41.

3. Liu WY, Wong CF, Chung KM, Jiang JW, Leung FC. Comparative genome analysis of Enterobacter cloacae. PLoS One. 2013;8, e74487.

4. Wisplinghoff $H$, Bischoff $T$, Tallent SM, Seifert $H$, Wenzel RP, Edmond MB. Nosocomial bloodstream infections in US hospitals: analysis of 24,179 cases from a prospective nationwide surveillance study. Clin Infect Dis. 2004;39:309-17.

5. Chen HN, Lee ML, Yu WK, Lin YW, Tsao LY. Late-onset Enterobacter cloacae sepsis in very-low-birth-weight neonates: experience in a medical center. Pediatr Neonatol. 2009;50:3-7.

6. Al-Hasan MN, Lahr BD, Eckel-Passow JE, Baddour LM. Temporal trends in Enterobacter species bloodstream infection: a population-based study from 1998-2007. Clin Microbiol Infect. 2011;17:539-45.

7. Harris PN, Ferguson JK. Antibiotic therapy for inducible AmpC betalactamase-producing Gram-negative bacilli: what are the alternatives to carbapenems, quinolones and aminoglycosides? Int J Antimicrob Agents. 2012;40:297-305.

8. Chow JW, Fine MJ, Shlaes DM, Quinn JP, Hooper DC, Johnson MP, Ramphal R, Wagener MM, Miyashiro DK, Yu VL. Enterobacter bacteremia: clinical features and emergence of antibiotic resistance during therapy. Ann Intern Med. 1991;115:585-90.

9. Kaye KS, Cosgrove S, Harris A, Eliopoulos GM, Carmeli Y. Risk factors for emergence of resistance to broad-spectrum cephalosporins among Enterobacter spp. Antimicrob Agents Chemother. 2001;45:2628-30.

10. Choi SH, Lee JE, Park SJ, Choi SH, Lee SO, Jeong JY, Kim MN, Woo JH, Kim YS. Emergence of antibiotic resistance during therapy for infections caused by Enterobacteriaceae producing AmpC beta-lactamase: implications for antibiotic use. Antimicrob Agents Chemother. 2008;52:995-1000.

11. Siebert JD, Thomson Jr RB, Tan JS, Gerson LW. Emergence of antimicrobial resistance in gram-negative bacilli causing bacteremia during therapy. Am J Clin Pathol. 1993;100:47-51.

12. Boyle RJ, Curtis N, Kelly N, Garland SM, Carapetis JR. Clinical implications of inducible beta-lactamase activity in Gram-negative bacteremia in children. Pediatr Infect Dis J. 2002;21:935-40.

13. Cosgrove SE, Kaye KS, Eliopoulous GM, Carmeli Y. Health and economic outcomes of the emergence of third-generation cephalosporin resistance in Enterobacter species. Arch Intern Med. 2002;162:185-90.

14. Qureshi ZA, Paterson DL, Pakstis DL, Adams-Haduch JM, Sandkovsky G, Sordillo E, Polsky B, Peleg AY, Bhussar MK, Doi Y. Risk factors and outcome of extended-spectrum beta-lactamase-producing Enterobacter cloacae bloodstream infections. Int J Antimicrob Agents. 2011;37:26-32.

15. Chen $\mathrm{CH}$, Huang CC. Risk factor analysis for extended-spectrum betalactamase-producing Enterobacter cloacae bloodstream infections in central Taiwan. BMC Infect Dis. 2013;13:417.

16. Wozniak A, Villagra NA, Undabarrena A, Gallardo N, Keller N, Moraga M, Roman JC, Mora GC, Garcia P. Porin alterations present in noncarbapenemase-producing Enterobacteriaceae with high and intermediate levels of carbapenem resistance in Chile. J Med Microbiol. 2012;61:1270-9.

17. Manzur A, Tubau F, Pujol M, Calatayud L, Dominguez MA, Pena C, Sora M, Gudiol F, Ariza J. Nosocomial outbreak due to extended-spectrum-betalactamase- producing Enterobacter cloacae in a cardiothoracic intensive care unit. J Clin Microbiol. 2007;45:2365-9.

18. Chang CL, Su LH, Lu CM, Tai FT, Huang YC, Chang KK. Outbreak of ertapenem-resistant Enterobacter cloacae urinary tract infections due to a contaminated ureteroscope. J Hosp Infect. 2013;85:118-24.

19. Cremet L, Bourigault C, Lepelletier D, Guillouzouic A, Juvin ME, Reynaud A Corvec S, Caroff N. Nosocomial outbreak of carbapenem-resistant
Enterobacter cloacae highlighting the interspecies transferability of the blaOXA-48 gene in the gut flora. J Antimicrob Chemother. 2012;67:1041-3.

20. Fernandez A, Pereira MJ, Suarez JM, Poza M, Trevino M, Villalon P, SaezNieto JA, Regueiro BJ, Villanueva R, Bou G. Emergence in Spain of a multidrug-resistant Enterobacter cloacae clinical isolate producing SFO-1 extended-spectrum beta-lactamase. J Clin Microbiol. 2011;49:822-8.

21. Haraoui LP, Levesque S, Lefebvre B, Blanchette R, Tomkinson M, Mataseje L, Mulvey MR, Miller MA. Polyclonal outbreak of KPC-3-producing Enterobacter cloacae at a single hospital in Montreal, Quebec, Canada. J Clin Microbiol. 2013;51:2406-8.

22. Boucher HW, Talbot GH, Bradley JS, Edwards JE, Gilbert D, Rice LB, Scheld M, Spellberg B, Bartlett J. Bad bugs, no drugs: no ESKAPE! An update from the Infectious Diseases Society of America. Clin Infect Dis. 2009;48:1-12.

23. Siedner MJ, Galar A, Guzman-Suarez BB, Kubiak DW, Baghdady N, Ferraro MJ, Hooper DC, O'Brien TF, Marty FM. Cefepime vs other antibacterial agents for the treatment of Enterobacter species bacteremia. Clin Infect Dis. 2014;58:1554-63.

24. Wolff MA, Young CL, Ramphal R. Antibiotic therapy for enterobacter meningitis: a retrospective review of 13 episodes and review of the literature. Clin Infect Dis. 1993;16:772-7.

25. Marcos M, Inurrieta A, Soriano A, Martinez JA, Almela M, Marco F, Mensa J. Effect of antimicrobial therapy on mortality in 377 episodes of Enterobacter spp. bacteraemia. J Antimicrob Chemother. 2008;62:397-403.

26. Le Gall JR, Neumann A, Hemery F, Bleriot JP, Fulgencio JP, Garrigues B, Gouzes C, Lepage E, Moine P, Villers D. Mortality prediction using SAPS II: an update for French intensive care units. Crit Care. 2005:9:R645-652

27. Implementation Guide for Surveillance of Central Line Associated Bloodstream Infection [http://www.safetyandquality.gov.au/wp-content/ uploads/2012/02/Implementation-guide-CLABSI-Consultation-EditionNovember-2011.pdf]. Accessed Nov 2016.

28. Auricht E, Borgert J, Butler M, Cadwallader H, Collignon P, Cooper C, Eades M, Ferguson J, Kampen R, Looke D, et al. Uniform national denominator definitions for infection control clinical indicators: surgical site and health care associated blood stream infection. Aust Infect Control. 2001;6:47-53.

29. CDC/NHSN Surveillance Definitions for Specific Types of Infections [http:// www.cdc.gov/nhsn/pdfs/pscmanual/17pscnosinfdef_current.pdf]. Accessed Nov 2016.

30. Antibiotic Expert Groups: Therapeutic guidelines: antibiotic. Version 15. Melbourne: Therapeutic Guidelines Limited; 2014.

31. Clinical and Laboratory Standards Institute. Performance standards for antimicrobial susceptibility testing; twenty-first informational supplement. In: CLSI document M100-S20. Wayne, PA: Clinical and Laboratory Standards Institute; 2011.

32. Breakpoint tables for interpretation of MICs and zone diameters version 5.0 [http://www.eucast.org/ast_of_bacteria/previous_versions_of_documents]. Accessed Nov 2015.

33. Jacoby GA. AmpC beta-lactamases. Clin Microbiol Rev. 2009;22:161-82.

34. Harris PN, Alder L, Paterson DL. Antimicrobial susceptibility reporting and treatment selection for AmpC-producing Enterobacteriaceae: what do microbiologists and infectious disease practitioners actually practice? Pathology. 2015;47:386-8.

35. Hombach M, Wolfensberger A, Kuster SP, Bottger EC. Influence of clinical breakpoint changes from CLSI 2009 to EUCAST 2011 antimicrobial susceptibility testing guidelines on multidrug resistance rates of Gramnegative rods. J Clin Microbiol. 2013;51:2385-7.

36. EUCAST guidelines for detection of resistance mechanisms and specific resistances of clinical and/or epidemiological importance [http://www. eucast.org/resistance_mechanisms/]. Accessed Nov 2016. 